\title{
EXPOSICIÓN VIRTUAL COMO HERRAMIENTA PARA EL TRATAMIENTO DEL TRASTORNO OBSESIVO COMPULSIVO
}

\author{
VIRTUAL EXHIBITION AS A TOOL FOR THE TREATMENT OF OBSESSIVE \\ COMPULSIVE DISORDER
}

Georgina Cárdenas L. ${ }^{1}$; Sandra Muñoz M.; Paola Oviedo L.

Facultad de Psicología de la Universidad Autónoma de México, México

(RECIBIDO 02/08/2012, ACEPTADO 25/11/2012)

\begin{abstract}
RESUMEN
El trastorno obsesivo compulsivo afecta al $2.6 \%$ de la población en México y es un una problemática que afecta severamente la calidad de vida de las personas que lo padecen, siendo clasificado por la OMS como la décima causa de discapacidad en el mundo, por lo que es un problema de salud que necesita atención por parte de los profesionales de la salud. Existen estudios sobre diversos tratamientos para este trastorno, entre ellos se encuentran el tratamiento de exposición y prevención de respuesta y el tratamiento cognitivo. El tratamiento puede ser percibido como aversivo y entre el 25 y el 35 por ciento de los pacientes abandonan el tratamiento debido a la ansiedad que experimentan por la exposición, el Laboratorio de Enseñanza Virtual y Ciberpsicología presenta un tratamiento basado en realidad virtual para poder exponer de forma controlada y menos aversiva a los pacientes obsesivo-compulsivos, el tratamiento se basa en la exposición y prevención de respuesta pero dando la facilidad al terapeuta de poder graduar de mejor forma los estímulos y la posibilidad de poder replicar los eventos la cantidad de veces que sea necesario. Por otra parte al paciente le permite exponerse en un ambiente virtual que le puede parecer seguro y que le permitirá hacer la exposición de forma gradual y menos aversiva. Los ambientes virtuales creados para dicho propósito son lugares contextualizados a la cultura mexicana y que están relacionados con la vida cotidiana del paciente para ayudar a éste a mejorar su calidad de vida así como reducir la evitación y ansiedad experimentada.
\end{abstract}

\begin{abstract}
In Mexico City $2.6 \%$ of population have Obsessive Compulsive Disorder, this is a that impact the quality of life in the persons who presents, it was classified by WHO like tenth disability cause in the world, it's for this reason that needs closer attention about mental health professionals, there are several studies about treatment for this disorder, between these we can find the exposure and response prevention treatment and cognitive treatment. The treatment could be perceived like aversive, and between 25 and 35 per cent of patients
\end{abstract}


drop out the treatment to be due to experimented anxiety during exposure, Virtual Teaching Laboratory presents avirtual reality based treatment for expose obsessive compulsive patients in a controlled way and lower aversive, this treatment it's based in exposure and response prevention, but give an advantage fortherapist, who can graduate in a better way the stimulus and have the possibility of reply the events the time it was necessary. Virtual environments developed for this purpose are places that are in context with Mexican culture and are related with dailylife for helps patient to improve his quality of life and reduce avoidance and anxiety scores.

\section{INTRODUCCIÓN}

En la población mundial el Trastorno Obsesivo Compulsivo (TOC) afecta entre el 2\% y 3\%, mientras que en México de acuerdo con la Encuesta Epidemiológica Psiquiátrica de 2003 se reporta un prevalencia total de un $2.6 \%$ con una edad media de inicio de 19 años, específicamente en la Ciudad de México la prevalencia es de $1.4 \%$ y la edad promedio de inicio entre hombres y mujeres fue de 22 años en un estudio realizado por Caraveo (2004). La comorbilidad que presenta este trastorno es mayor con el abuso de sustancias en el caso de los hombres y en el caso de las mujeres existe mayor comorbilidad con las fobias específicas y el episodio depresivo.

El TOC tiene un impacto negativo en la calidad de vida de los pacientes influyendo en su vida familiar y social (Pérez, Olson, \& Horton, 2007), de hecho de acuerdo a la OMS y el Banco Mundial está clasificado como la décima causa de discapacidad en el mundo. Este trastorno presenta una mayor comorbilidad con el abuso de alcohol en el caso de los hombres, precediendo el abuso de la sustancia al TOC mientras que en el caso de las mujeres la comorbilidad es mayor con el episodio depresivo, las fobias específicas y otros trastornos de ansiedad, de los cuales la fobia específica y la ansiedad generalizada preceden en la aparición de los síntomas al trastorno obsesivo y la fobia social y la agorafobia aparecen después del TOC.

De los pacientes con trastorno obsesivo compulsivo en México, solo el ocho por ciento de los casos busca ayuda, y es más frecuente que aquellos pacientes que no presentan comorbilidad no busquen opción de tratamiento.

Tomando en cuenta la epidemiología y la gravedad de los síntomas que afectan la vida diaria del paciente hasta incapacitarlo en áreas como el trabajo, las relaciones sociales y la escuela, principalmente, el Laboratorio de Enseñanza Virtual y Ciberpsicología desarrolló ambientes virtuales para el tratamiento del Trastorno Obsesivo Compulsivo para ayudar a los pacientes a enfrentar situaciones que les causan ansiedad, reducir las conductas compulsivas así como los pensamientos intrusivos y obsesivos.

\section{El Trastorno Obsesivo Compulsivo. Conceptualización y Tratamiento}

La conceptualización, evaluación, clasificación y tratamiento del Trastorno Obsesivo Compulsivo (TOC) ha sufrido cambios radicales en los últimos años, sobre todo desde el surgimiento del tratamiento farmacológico con antidepresivos 
tricíclicos y actualmente Inhibidores Selectivos de Recaptura de Serotonina (ISRS) el cual se basa en la premisa de que existen ciertas anormalidades anatómicas y funcionales en el sistema nervioso de los pacientes obsesivos. Todo este cambio ha significado un nuevo campo de investigación sobre un trastorno que había sido poco estudiado y se consideraba como uno de los más resistentes al tratamiento por su complejidad y heterogeneidad inter e intra-sujeto.

Woody\&Sanderson (1998), marcan el tratamiento de exposición y prevención de respuesta (EPR) como un tratamiento empíricamente validado basados en una actualización de los tratamientos que han obtenido eficacia. Esta terapia se basa en la teoría del aprendizaje por lo cual contiene un componente de exposición para provocar las obsesiones y la prevención o neutralización de las respuestas rituales que reducen la ansiedad.

Por otra parte la Terapia Cognitiva (TC) que también ha resultado eficaz para el tratamiento del TOC se basa en la teoría cognitiva de los trastornos emocionales propuesta por Beck y el modelo propuesto por Salkovskis en 1985, que plantea que los pensamientos intrusivos se convierten en obsesiones clínicas cuando los individuos evalúan dichas intrusiones como un indicador de que pueden ser responsables del daño que pueden ocasionar a otros o a ellos mismos, por lo que toman una acción para prevenir ese desastre. La TC intenta modificar las evaluaciones de responsabilidad excesiva y las conductas neutralizantes que mantienen las creencias de que la persona es responsable de prevenir el daño.

Desde hace algunos años se maneja otra opción de tratamiento que representa la combinación de las técnicas conductuales de exposición y prevención de respuesta introducidas por Meyer en 1966, y de los estudios en el campo cognitivo representados principalmente por el Grupo de Trabajo sobre Cogniciones Obsesivo Compulsivas (Obsessive Compulsive Cognition Working Group, OCCWG) han dado origen al Tratamiento Cognitivo-Conductual (TCC) con el cual se logra reducir entre el 60 y 70 por ciento de síntomas tales como: conductas compulsivas, estrategias neutralizadoras, creencias disfuncionales, pensamientos intrusivos demostrando la efectividad clínica (Abramowitz, 2007); además de ser el único tratamiento psicológico que da cuenta de cambios observables (gracias a técnicas de neuroimagen) a nivel sistema nervioso. Ha sido tal el impacto de la eficacia de la TCC que actualmente se le considera el tratamiento de elección para el TOC. Sin embargo, el tratamiento puede percibirse como aversivo debido a que está basado en la exposición prolongada a estímulos temidos o causantes de malestar, y al menos $25 \%$ a $30 \%$ que buscan ayuda psicológica rehúsan este tipo de intervención o la abandonan de manera prematura (Abramowitz, 1998, 2006; Foa \& Kozak, 1986; Franklin, Abramowitz, Kozak, Levitt, \& Foa, 2000), debido al temor y ansiedad que experimentan al cesar la realización de sus rituales, estrategias de neutralización o conductas compulsivas debido a la creencia de que las consecuencias temidas pueden cumplirse. Por ello, se pensó en una variación a este tratamiento de tal forma que el impacto negativo se viera disminuido. Una herramienta alternativa viable para dichos fines se ha encontrado en el campo de 
las Tecnologías de la Información y comunicación (TIC) las cuales han avanzado a un ritmo acelerado en las últimas décadas. Puede observarse su alcance en diversas áreas tanto científicas como aplicadas debido a su adaptabilidad a diversos contextos.

\section{Inclusión de la Realidad Virtual al Tratamiento Psicológico}

En el campo específico de la tecnología multimedia ha habido grandes avances y uno de ellos lo constituye el desarrollo de la realidad virtual (RV) que de acuerdo a Burdea en 1994 es definida como una interface humano-computadora que involucra una simulación en tiempo real y una interacción a través de múltiples canales sensoriales (1994). El uso de esta tecnología se basa en no exponer directamente a los sujetos a situaciones temidas, que pudieran resultar costosas, incómodas o consideradas inaccesibles en ciertas circunstancias, sino hacerlo de manera simulada. Desde su surgimiento fue de gran ayuda en servicios, telecomunicaciones, educación, entretenimiento, ingenieríay otros campos de aplicación y generación de conocimiento.

En el área de la salud se han observado aplicaciones de la RV en ámbitos tales como: rehabilitación de personas con infartos cerebrales, entrenamiento de cirujanos, como distracción con pacientes quemados y la aplicación para el tratamiento de trastornos mentales con eficiencia.

En el campo específico de la psicología, fueron conducidos en 1996, por Rothbaum y colaboradores, los primeros experimentos de exposición con realidad virtual, aunque la tecnología era aún incipiente. Desde entonces, el número de estudios que evalúan la efectividad de la terapia de exposición con realidad virtual se han incrementado rápidamente, reforzados por el progreso en tecnologías computacionales, que ahora son más sofisticadas y tienen mayores posibilidades para el Tratamiento con Realidad Virtual. La mayor parte de la investigación se ha centrado en los trastornos de ansiedad, aunque dentro de las posibilidades de investigación se encuentra el tratamiento con realidad virtual para los trastornos de alimentación, adicciones, evaluación psicológica, control del dolor, cuidados paliativos, algunos trastornos del control de los impulsos y rehabilitación (Côté \& Bouchard, 2005; Emmelkamp, 2005; North, North, \& Coble, 1998). Existen varios estudios que apoyan la evidencia de que la exposición con realidad virtual es eficaz para el tratamiento de las fobias y otros trastornos de ansiedad como el miedo a volar, acrofobia, agorafobia, miedo a hablar en público.

la terapia de exposición con realidad virtual hace uso de ambientes virtuales que son inmersivos y provocan ansiedad suficiente para que el paciente pueda trabajar y afrontarlos. La exposición se lleva cabo de forma prolongada al igual que la exposición en vivo para que la extinción de la conducta ocurra, sin embargo, no es necesario llevar al paciente a cada una de las situaciones temidas para que esto suceda, el clínico puede trabajar desde su consultorio.

De acuerdo con la teoría de Foa y Kozak (1986), hay tres condiciones para que la Terapia de Exposición con realidad virtual sea efectiva. Primero, los pacientes 
necesitan sentirse presentes en los ambientes virtuales para lograr experimentar totalmente el ambiente como un lugar visitado. En segundo lugar, el ambiente debe ser capaz de provocar emociones, de otra forma, la extinción no ocurrirá. Finalmente, la extinción y co-ocurrencia de cambios cognitivos tienen que generalizarse a las situaciones reales de tal forma que esas situaciones de la vida real no serán evitadas nunca más o sean afrontadas con un menor monto de ansiedad.

Para que los pacientes se sirven dentro del escenario virtual, de acuerdo con Burdea (1994) deben existir tres condiciones: la inmersión, interacción e imaginación, lo cual hace evidente que para que la experiencia de presencia se dé se necesita tanto de aspectos técnicos que incluyen el hardware y el software, como del usuario que necesita incluir la imaginación dentro de la experiencia, además de los aspectos que son inherentes al usuario como el campo visual, la agudeza visual, la concentración y atención.

La terapia de exposición con realidad virtual está basada en la suposición de que las personas se sienten "presentes" en el ambiente virtual. La presencia es entonces asumida como una variable mediadora importante y es definida como "El estado psicológico o percepción subjetiva en que aunque parte o toda la experiencia individual en curso es generada por/y/o filtrada a través de tecnología hecha por humanos, parte o toda la percepción del individuo falla en el reconocimiento exacto de la tecnología en la experiencia”. Excepto en los casos más extremos, el individuo puede indicar correctamente que está usando la tecnología, pero en cierto nivel y en cierto grado, sus percepciones pasan por alto ese conocimiento y esos objetos, eventos, entidades y ambientes son percibidos como si la tecnología no estuviera involucrada en la experiencia. La presencia se puede medir desde diversos puntos de vista, por ejemplo, utilizando una evaluación continua de presencia donde el usuario a través de una palanca de diferentes posiciones puede indicar el nivel de presencia, sin embargo, estas mediciones resultan poco funcionales ya que el usuario necesita siempre una mano para el dispositivo de calificación y lo distrae de la inmersión. Otro tipo de mediciones pueden ser de tipo fisiológico en donde se pueden recabar datos acerca de los diferentes niveles de inmersión de acuerdo a los cambios en los patrones de las respuestas fisiológicas. Finalmente, se han hecho escalas o cuestionarios que tienen la ventaja de no ser invasivos y no interrumpir la experiencia de inmersión, y se pueden obtener datos sobre la sensación de presencia. Los primeros esfuerzos par realizar este tipo de mediciones se hicieron por Slater en el año 1994 y por Witmer y Singer en 1998, por otra parte en el año 2000 Baños y colaboradores desarrollaron un cuestionario que medía presencia y juicio de realidad.

Se han llevado a cabo estudios de evaluación basado en tecnologías de la información para el Trastorno Obsesivo-Compulsivo (Kirkby, 2000; Van den Hout, 2003, 2004). Un ejemplo de ello es el estudio de Clark (1998) en el cual se realizaron experimentos conductuales simulados con realidad virtual centrándose específicamente en la conducta de lavado compulsivo. La finalidad era exponer al paciente a un ambiente virtual con suciedad excesiva con la cual el paciente debía estar en contacto de tal forma que le provocara el impulso para lavarse. 
Conforme la exposición continuaba ante un ambiente cada vez más contaminado, el debía reducir gradualmente su conducta de lavado. Los resultados de este estudio fueron medianamente satisfactorios y los autores plantearon la posibilidad de ver los experimentos conductuales con realidad virtual como un apoyo inicial o pre-entrenamiento y se consideraba que no podría sustituir a la terapia tradicional basada en exposición en vivo y prevención de respuesta, al tratarse el TOC de un trastorno heterogéneo, que varía en presentación de la sintomatología entre un paciente y otro, por lo cual los autores concluyeron que la realidad virtual sería una herramienta inicial útil en conjunto con la psicoeducación para ayudar a la justificación del tratamiento (Krijn, Emmelkamp, Olafsson, \& Biemond, 2004). Debe tomarse en consideración que los estudios mencionados acerca de la realidad virtual como herramienta terapéutica para el tratamiento del TOC presentaban ciertas limitaciones técnicas que dificultaban la inmersión de los pacientes, pues se trataba de simulaciones pobres en relación a los gráficos y situaciones disponibles a las que se podría exponer el paciente.

La potencialidad de la realidad virtual como herramienta terapéutica para el TOC se ha considerado, por las características que presenta esta tecnología, ya que posee la ventajas de que el terapeuta puede modificar diversas variables que en situaciones de la vida cotidiana serían difíciles de manejar y controlar, además de ser ambientes menos aversivos para pacientes que perciben la terapia de exposición en vivo como algo demasiado difícil de afrontar; además de la eficacia observada en el tratamiento de otras problemáticas utilizando la exposición virtual.

Por lo anterior, el Laboratorio de Enseñanza Virtual y Ciberpsicología de la Facultad de Psicología de la UNAM junto con el Observatorio de Visualización Ixtli han trabajado en un proyecto relacionado con la creación de ambientes virtuales que servirán como apoyo al tratamiento del Trastorno Obsesivo Compulsivo específicamente para dos subtipos de este trastorno identificados en la literatura clínica como lavado y orden. Este tipo de intervención tiene como finalidad reducir la aversión y excesiva angustia que significa para los pacientes el comprometerse en un tratamiento basado en exposición y prevención de respuesta tradicional, pues a diferencia de este último, en el tratamiento apoyado con realidad virtual se expondría al paciente a estímulos desencadenantes de los temores obsesivos en ambientes gráficos creados por computadora, de tal forma que los beneficios obtenidos con este tipo de tratamiento son entre otros: reducción de la excesiva angustia y sufrimiento que experimentan los pacientes al someterse a este tratamiento, la confidencialidad, reducción en tiempo y coste económico.

\section{Propuesta de tratamiento con realidad virtual}

Los ambientes virtuales desarrollados para estos fines son un autobús, una habitación, una fonda mexicana y un baño público. Cada uno de los escenarios fue desarrollado con la finalidad de incluir elementos desencadenantes que un paciente puede encontrar en la vida cotidiana y que estén contextualizados a su cultura, de tal forma que se puede exponer a los pacientes a dichos ambientes y poder controlar las variables dentro del entorno. Las variables o elementos incluidos se 
elaboraron con la finalidad de que fungieran como desencadenantes de miedos obsesivos particulares: contaminación, desorden y simetría. Las obsesiones de contaminación se han identificado como el subtipo de TOC más frecuente, pues está presente en alrededor de $70 \%$ de los pacientes con TOC (Eddy, 2004). Este subtipo consiste principalmente en un temor excesivo e irracional a contaminarse por suciedad, gérmenes, diversas sustancias y los desencadenantes más frecuentes de estas obsesiones suelen ser estímulos externos (muebles, pisos, plantas, chapas de las puertas, jeringas, barandales, tocar a otras personas, etc). Por otro lado, las obsesiones de simetría y orden se centran en la necesidad de tener objetos o eventos en determinado orden, posición o simetría por temor a que ocurran consecuencias catastróficas o el malestar y extrema incomodidad que experimentan cuando las cosas no están en el orden correcto.

Los ambientes virtuales son parte de un protocolo de tratamiento que incluye la prevención de respuesta y otras técnicas como la demora del pensamiento, la respiración controlada, las autoafirmaciones positivas y la reestructuración cognitiva, constituyéndose como un tratamiento integral en el cual una de las partes medulares sería la exposición virtual a los ambientes virtuales elaborados.

\section{Autobús}

$\mathrm{Al}$ ser un espacio público, se considera un escenario en donde las personas tienen que interactuar necesariamente con los elementos que lo componen, tales como personas y elementos inherentes a dicho espacio. Para provocar algunos miedos obsesivos, este escenario contiene estímulos que suelen ser aversivos para los pacientes con TOC con obsesiones de contaminación, dichos estímulos se relacionan con suciedad en los asientos, en los tubos para asirse, en el piso y personas sucias o desaliñadas o que en apariencia padezcan alguna enfermedad, además de personal de salud, enfermeras o médicos que se relacionan con hospitales y personas enfermas a quienes los pacientes perciben como una fuente importante de infección.

\section{Fonda tradicional}

Representa un lugar público de consumo de alimentos, por ello se considera un escenario idóneo para trabajar con obsesiones de contaminación pues la persona con TOC no puede estar segura de las condiciones en que se preparan sus alimentos con relación a higiene en el manejo de los alimentos por parte de otras personas o procedencia de los ingredientes con que se preparan los platillos; la limpieza de los elementos que componen el lugar (trastes, cubiertos, charolas, mesas, asientos, teléfonos, piso) por el uso que gran cantidad de personas hacen de estos. De igual forma contiene elementos que pueden ayudar a trabajar con las obsesiones de orden, simetría, incompletitud y exactitud tales como las salsas, saleros, jarras servilleteros, y colocación del servicio que se hallan en desorden para provocar ansiedad, malestar o incomodidad al paciente.

\section{Habitación}

Este escenario se compone de elementos con los que el paciente puede estar familiarizado por su uso frecuente en la vida cotidiana, ello lo hace un ambiente 
adecuado para trabajar tanto obsesiones de contaminación, como obsesiones de orden y simetría.

El escenario de la habitación está compuesto por una cama (con sábana, colcha, edredones, rodillos), un librero (con televisión, libros), un escritorio (con artículos para trabajo de oficina tales como plumas cuadernos, hojas), cómodas con diversos artículos dentro (medicinas, revistas, etc.) y un ropero (con ropa, zapatos y otros accesorios dentro). Es un espacio en que el paciente puede estar expuesto a diversos grados de desorden de los elementos antes mencionados, así como a potencial contaminación por manchas, polvo, además de ser un espacio que hace referencia a su vida cotidiana.

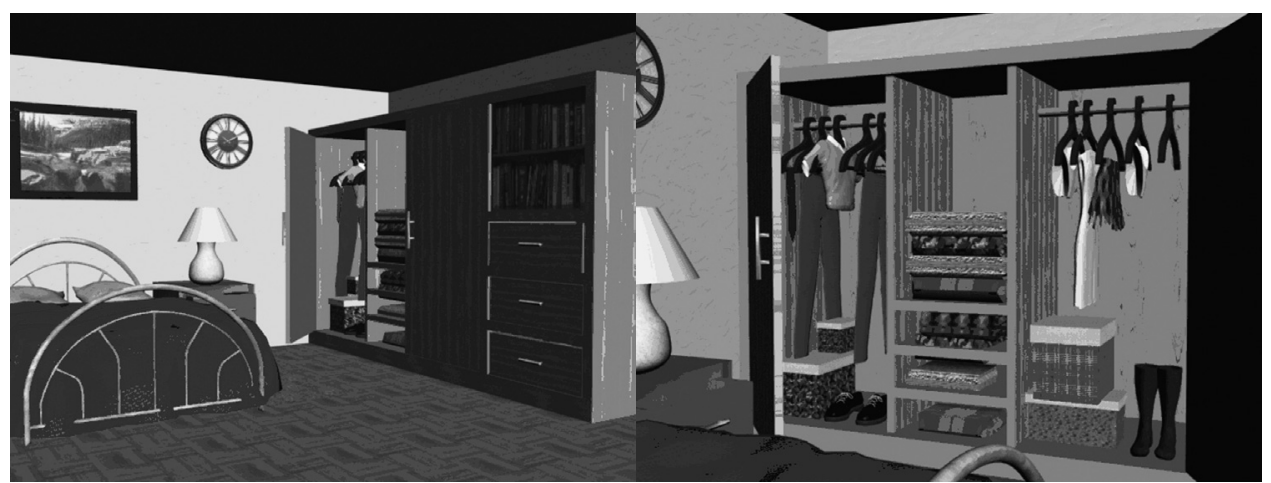

\section{Baño público}

Este escenario representa uno de los lugares más temidos por las personas que padecen obsesiones de contaminación al considerarte una fuente potencial de enfermedades, por el uso constante de gran cantidad de personas, en donde la suciedad percibida puede consistir mayormente en secreciones corporales (orina, heces, sangre, entre otras) que pueden ser percibidas como desagradables, contaminantes y medios de transmisión de enfermedades de diverso tipo. Todos los elementos que componen el lugar tienen como finalidad provocar malestar, incomodidad o ansiedad en el paciente por la contaminación que representan dichos estímulos.

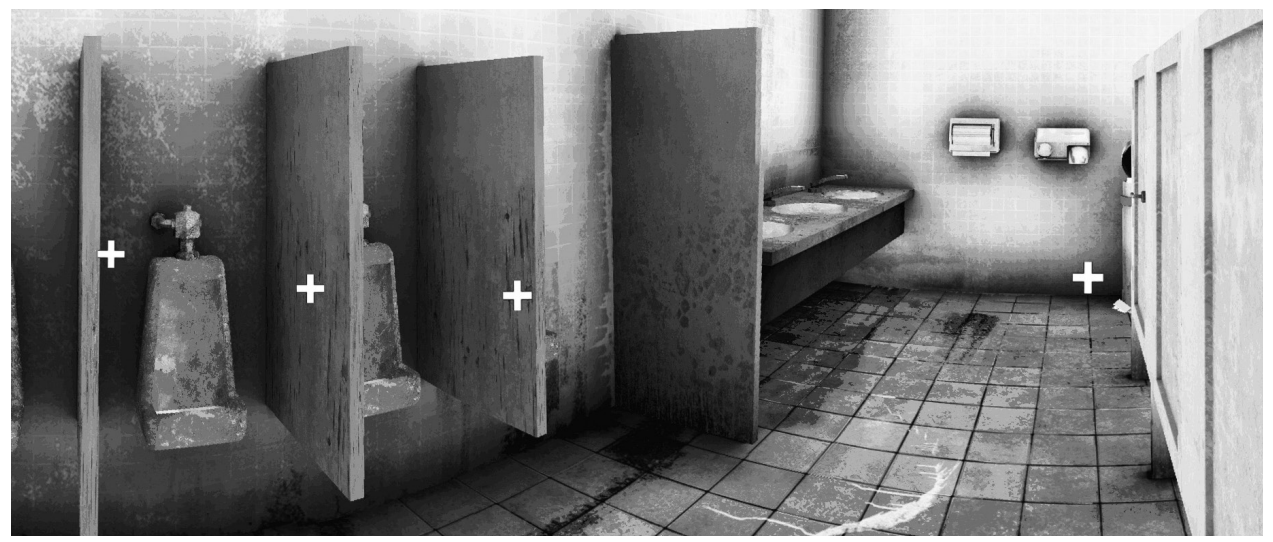


La introducción de las nuevas tecnologías en el tratamiento psicológico ha sido exitosa como se ha abordado a lo largo del documento; sin embargo, existen ciertos trastornos en los que se ha aplicado, en el caso del trastorno obsesivo compulsivo existen pocos estudios al respecto, por lo cual esta herramienta tecnológica desarrollada brinda algunas ventajas para los clínicos interesados en el tratamiento del Trastorno obsesivo compulsivo. El programa de realidad virtual aquí presentado muestra varias ventajas para los psicólogos clínicos que podrán realizar el tratamiento sin salir del consultorio recreando situaciones cotidianas, difíciles de encontrar dentro de la realidad, además los terapeutas podrán tener un mejor control de los estímulos al exponer al paciente a los escenarios; en cuanto al paciente el exponerse en los escenarios virtuales le resulta menos aversivo y podrá darse cuenta de que los pensamientos negativos y sobrevalorados que presenta no ocurren como él pensaba. También esta herramienta puede ser útil para la evaluación, que complementada con una lista, nos permitiría observar dentro del consultorio de forma presencial las compulsiones y las obsesiones para evaluar la severidad de las mismas y poder trabajar con ellas durante el tratamiento.

\section{REFERENCIAS BIBLIOGRÁFICAS}

Abramowitz, J. S. (1998). Does Cognitive-Behavioral Therapy Cure Obsessive-Compulsive Disorder? A Meta-Analytic Evaluation of Clinical Significance Behavior Therapy 29, 339-355.

Abramowitz, J. S. (2006). The Psychological Treatment of Obsessive-Compulsive Disorder. The Canadian Journal of Psychiatry, 51(7), 407-416.

Abramowitz, J. S. (2007). Trastorno Obsesivo-compulsivo México: Manual Moderno.

Baños, R., Botella, C., García Palacios, A., Villa, H., Perpina, C., \& Alcañiz, M. (2000). Presnece and Reality Juddment in Virtual Environments: A Unitary Construct. Cyberpsychology \& Behavior, 3(3), 327-335.

Burdea, G., \& Coiffet, P. (1994). Virtual Reality Technology. New York, USA: John Wiley \& Sons.

Caraveo-Anduaga, J., \& Colmenares, E. (2004). The Epimemiology of ObsessiveCompulsive Disorder in Mexico City. Salud Mental, 27(2), 1-6.

Clark, A., Kirkby, K.C., Daniels, B.A., Marks, I. M. (1998). A pilot study of computeraided vicarious exposure for obsessive-compulsive disorder. Australian and New Zealand Journal of Psychiatry, 32, 268-275.

Côté, S., \& Bouchard, S. (2005). Documenting the efficacy of virtual reality exposure with psychophysiological and information processing measures. Applied psychophysiology and biofeedback, 30(3), 217-232.

Eddy, K. T., Dutraa, L, Bradleyb, R., Westenb, D. (2004). A multidimensional metaanalysis of psychotherapy and pharmacotherapy for obsessive-compulsive disorder. Clinical Psychology Review, 24, 1011-1030. 
Emmelkamp, P. (2005). Innovations in clinical assessment and psychotherapy. Psychotherapy and psychosomatics, 74, 336-343.

Foa, E., \& Kozak, M. (1986). Emotional Processing of Fear: Exposure to Corrective Information. Psychological Bulletin, 99(1), 20-35.

Franklin, M., Abramowitz, J. S., Kozak, M., Levitt, J., \& Foa, E. (2000). Effectiveness of exposure and ritual prevention for obsessive-compulsive dosorder randomized compared with nonrandomized samples. Journal of Consulting and Clinical Psychology, 68(4), 594-602.

Kirkby, K. C., Berrios, G. E., Daniels, B.A., Menzies, R.G., Clark, A., Romano, A. (2000). Process-Outcome analysis in computer-aided treatment of obsessivecompulsive disorder. Comprehensive Psychiatry, 41(4), 259-265.

Krijn, M., Emmelkamp, P., Olafsson, R. P., \& Biemond, R. (2004). Virtual reality exposure therapy of anxiety disorders: A review. Clinical Psychology Review, 24, 259-281.

Medina-Mora, M. E., Borges, G., Lara, C., Benjet, C., Blanco, J., Fleiz, C., et al. (2003). Prevalencia de trastornos mentales y uso de servicios: resultados de la encuesta nacional de epidemiología psiquiátrica en México. Salud Mental, 26(4), 1-15.

North, M. M., North, S. M., \& Coble, J. R. (1998). Virtual reeality therapy. An effective treatment for phobias. In G. Riva, B. Wiederhold \& E. Molinari (Eds.), Virtual environments in clinical psychology and neuroscience. Amsterdam: IOS Press.

Pérez, O., Olson, T., \& Horton, K. (2007). Trastorno Obsesivo Compulsivo en la Frontera México-Estados Unidos. Revista Científica Electrónica de Psicología (3), 238-250.

Van den Hout, M. K. M. (2003). Repeated checking causes memory distrust. Research and Therapy, 41(3), 301-316.

Van den Hout, M. K. M. (2004). Obsessive-compulsive disorder and the paradoxical effects of perseverative behaviour on experienced uncertainty. Journal of Behavior Therapy, 35(2004), 165-181.

Woody, S., \& Sanderson, W. C. (1998). Manuals for empirically supported treatments:1998 update. Clininical Psychology, 51(1), 17-21. 